\title{
Cooperative Control for Uncertain Multiagent Systems via Distributed Output Regulation
}

\author{
Lu Yu and Jinzhi Wang \\ State Key Laboratory for Turbulence and Complex Systems, Department of Mechanics and Aerospace Engineering, \\ College of Engineering, Peking University, Beijing 100871, China
}

Correspondence should be addressed to Jinzhi Wang; jinzhiw@pku.edu.cn

Received 17 April 2013; Accepted 29 April 2013

Academic Editor: Guanghui Wen

Copyright (c) 2013 L. Yu and J. Wang. This is an open access article distributed under the Creative Commons Attribution License, which permits unrestricted use, distribution, and reproduction in any medium, provided the original work is properly cited.

The distributed robust output regulation problem for multiagent systems is considered. For heterogeneous uncertain linear systems and a linear exosystem, the controlling aim is to stabilize the closed-loop system and meanwhile let the regulated outputs converge to the origin asymptotically, by the help of local interaction. The communication topology considered is directed acyclic graphs, which means directed graphs without loops. With distributed dynamic state feedback controller and output feedback controller, respectively, the solvability of the problem and the algorithm of controller design are both investigated. The solvability conditions are given in terms of linear matrix inequalities (LMIs). It is shown that, for polytopic uncertainties, the distributed controllers constructed by solving LMIs can satisfy the requirements of output regulation property.

\section{Introduction}

Recently, there are amounts of researches on cooperative control for multiagent systems (MASs) because of broad applications. An MAS is a practical model to describe dynamic agents which can exchange information by communication, such as unmanned air vehicles and sensor networks. According to different control objectives, problems of consensus, tracking, formation, flocking and the rest have been widely studied.

Among those cooperative control problems, the consensus problem and the tracking problem share some common characteristics. The consensus problem requires the MAS to reach an agreement by protocols based on local information. In $[1,2]$, the consensus problem is primarily studied, and the basic problem framework is formed. The consensus problem has been investigated for different kinds of agents, such as first-order integrators in [3], second-order integrators in [4, 5], linear systems in [6-8], and nonlinear systems in [9-12]. Recently, the output consensus problem for heterogeneous systems also attracted researchers. The dynamics and even dimensions of the agents are possibly different, so it is desirable to focus on the synchronization of outputs. In [13], the consensus of a class of second-order integrators with unknown nonlinear dynamics is considered. As for high-order systems, the frequency domain approach is used to discuss the consensus of heterogenous linear systems in $[14,15]$. Uncertain minimum-phase linear MASs are studied in [16], by a low-gain approach. In [7], general uncertain linear MASs are considered, and a sufficient and necessary condition for the solvability of the output consensus problem is proposed. It is admirable that Wieland et al. introduce an important concept of internal model to cooperative control, which is also fundamental in output regulation theory.

For the leader-follower consensus problem, also called consensus tracking problem, it involves one or multiple leaders and several followers. A leader is usually the target to be tracked, or the agent that directly receives the information of the target. Distributed controllers are designed to help all the agents to track one or multiple leaders by cooperation. The tracking problem for MASs has been studied in a lot of papers, such as [17-22]. Note that for heterogenous MASs, the outputs of all the agents are required to be synchronized in the issue of both consensus problem and tracking problem. To consider the two kinds of problems under a unified framework is one of the motivations to introduce distributed output regulation (DOR) problem. 
According to [23], output regulation problem involves an exosystem and a regulated output defined by a combination of the measurement output and the output of the exosystem. Controllers are designed to stabilize the closed-loop system and modulate the regulated outputs to the origin. So, it has an attracting performance on solving a tracking problem in the presence of disturbances. The classic output regulation theory cannot directly be applied to MASs nevertheless. Actually the controllers obtained are probably not in a distributed form. So, a framework of DOR for MASs is introduced in [2426]. What is mainly different from the classic theory is that the controllers have to be distributed, and only local information is available. In [24], homogenous linear MASs and a directed topology are considered, and dynamic state/output feedback controllers are designed. In [25], heterogenous MASs are challenged, and effective controllers are obtained under the directed acyclic topology. Different from the two works above, the limits on topology are dispelled in [26] by reconstructing the form of controllers. The communication of relative states of controllers replaces the communication of relative outputs.

This paper is basically motivated by [25]. In [25], the distributed controllers are robust to uncertain dynamics with sufficiently small uncertainties. However, more analyses based on information of the uncertainties are not involved in [25]. What we focus on in our paper is that how to design robust controllers if the uncertainty is structured. We suppose that the uncertainties are in a polytopic form. For the distributed robust output regulation problem, we give sufficient conditions of the solvability in terms of linear matrix inequalities (LMIs) and present an approach of controllers design.

An outline of this paper is as follows. In Section 2, some preliminaries and the problem statement are given. In Section 3, for distributed output regulation problem with polytopic uncertainties, the sufficient conditions of solvability and algorithms of both state and output feedback controllers design are proposed. In Section 4, a practical example is taken to show the control effect of our approach, which is compared with that of the algorithm given in [25]. In Section 5, a conclusion is given.

The following notations will be used throughout this paper. $\mathbb{R}$ is the set of real numbers. $I_{n}$ is the $n$-dimensional identity matrix. $0_{m \times n}$ is the zero matrix with $m$ rows and $n$ columns, and 0 is the zero matrix with appropriate dimensions. For a symmetric matrix $M \in \mathbb{R}^{n \times n}, M>0$ which means that $M$ is positive definite. $M^{T}$ is the transposition of the matrix $M$. diag block $\left(M_{1}, \ldots, M_{p}\right)$ means a block diagonal matrix with $M_{1}, \ldots, M_{p}$ as the diagonal entries. $\otimes$ denotes the Kronecker product.

\section{Problem Statement}

Let us begin with notations in graph theory [27]. A graph is denoted by $\mathscr{G}=(\mathscr{V}, \mathscr{E})$, where $\mathscr{V}=\left\{v_{1}, v_{2}, \ldots, v_{N}\right\}$ is the set of $N$ nodes and $\mathscr{E} \subseteq \mathscr{V} \times \mathscr{V}$ is the set of edges. An edge from node $v_{j}$ to node $v_{i}$ is denoted by $(i, j) \in \mathscr{E}$. A subset of $\mathscr{E}\left\{\left(i_{1}, i_{2}\right),\left(i_{2}, i_{3}\right), \ldots,\left(i_{k}, i_{k+1}\right)\right\}$ is called a path from $v_{i_{k+1}}$ to $v_{i_{1}}$. If $i_{1}=i_{k+1}$, the path is called a loop. $N_{i}$ denotes the neighbor set $\{j:(i, j) \in \mathscr{E}, 1 \leq j \leq N\}$, whose cardinality is $\left|N_{i}\right|$. A constant matrix $A_{d}=\left[a_{i j}\right] \in \mathbb{R}^{N \times N}$ is called the adjacency matrix of graph $\mathscr{G}$ if $a_{i j}=1 /\left|N_{i}\right|$ when $(i, j) \in \mathscr{E}$ and $a_{i j}=0$ when $(i, j) \notin \mathscr{E}$. And a constant matrix $L=\left[l_{i j}\right]=I_{N}-A_{d} \in$ $\mathbb{R}^{N \times N}$ is called the Laplacian matrix of graph $\mathscr{G}$. A graph is called an undirected graph if for all $1 \leq i, j \leq N, a_{i j}=a_{j i}$. Or else, it is called a directed graph. If a directed graph does not contain a loop, it is called a directed acyclic graph.

Consider an exosystem with $\omega \in \mathbb{R}^{q}$ as the state, whose dynamics can be described as follows:

$$
\dot{\omega}(t)=S \omega(t),
$$

where $S \in \mathbb{R}^{q \times q}$ is a constant matrix, satisfying the following assumption as that in $[23,25]$.

Assumption 1. S has no eigenvalues with negative real parts.

A MAS consists of $N$ nonidentical dynamic agents which can exchange information among neighborhood. For $i=$ $1, \ldots, N$, the $i$ th agent can be expressed by

$$
\begin{gathered}
\dot{x}_{i}(t)=\bar{A}_{i} x_{i}(t)+\bar{B}_{i} u_{i}(t)+\bar{E}_{i} \omega(t), \\
y_{i}(t)=\bar{C}_{i} x_{i}(t), \\
e_{i}(t)=\bar{C}_{i} x_{i}(t)+F \omega(t),
\end{gathered}
$$

where $x_{i} \in \mathbb{R}^{p_{i}}, u_{i} \in \mathbb{R}^{m_{i}}, y_{i} \in \mathbb{R}^{n}$, and $e_{i} \in \mathbb{R}^{n}$ are, respectively, the state, the control input, the measurement output, and the regulated output of the $i$ th agent. $F \in \mathbb{R}^{n \times q}$ is a certain constant matrix, while those matrices $\bar{A}_{i} \in$ $\mathbb{R}^{p_{i} \times p_{i}}, \bar{B}_{i} \in \mathbb{R}^{p_{i} \times m_{i}}$, and $\bar{C}_{i} \in \mathbb{R}^{n \times p_{i}}$ are uncertain matrices represented as

$$
\begin{aligned}
& \bar{A}_{i}=\sum_{k=1}^{g_{i}} \lambda_{k i} A_{k i}, \\
& \bar{B}_{i}=\sum_{k=1}^{g_{i}} \lambda_{k i} B_{k i}, \\
& \bar{C}_{i}=\sum_{k=1}^{g_{i}} \lambda_{k i} C_{k i},
\end{aligned}
$$

where $A_{k i}, B_{k i}$, and $C_{k i}$ are known constant matrices, and $\lambda_{k i}$ are nonnegative constants satisfying that $\sum_{k=1}^{g_{i}} \lambda_{k i}=1$, for $i=1, \ldots, N, k=1, \ldots, g_{i} . \bar{E}_{i} \in \mathbb{R}^{p_{i} \times q}$ is an arbitrary constant matrix.

The controlling aim is to stabilize the closed-loop system and also to regulate $e_{1}, \ldots, e_{N}$ to the origin. For $i=1, \ldots, N$, if $e_{i}$ is available to the $i$ th agent, the output regulation of the $i$ th agent is simple to be achieved by classic output regulation theory. However, only some of the agents can get information of their own regulated outputs, which are called leader nodes. The set of their serial numbers is denoted by $\mathscr{L}$, while other agents utilize the relative output among neighbors to accomplish the output regulation property. To ensure that all the agents can receive the information of the exosystem by local interaction, the communication graph of the MAS satisfies the following assumption. 
Assumption 2. Graph $\mathscr{G}$ is a directed acyclic graph. And for each nonleader node $v_{i}$, there exists a leader node $v_{j}$ such that a path from node $v_{j}$ to node $v_{i}$ exists.

In this note, two kinds of distributed dynamic feedback controllers are considered.

(I) Distributed dynamic state feedback controller:

$$
\begin{gathered}
\dot{z}_{i}(t)=G_{1} z_{i}(t)+G_{2} e_{i \omega}(t), \\
u_{i}(t)=K_{1 i} x_{i}(t)+K_{2 i} z_{i}(t), \\
z_{i}(t) \in \mathbb{R}^{c}, \quad q \leq c, \quad i=1, \ldots, N,
\end{gathered}
$$

where

$$
e_{i \omega}(t)= \begin{cases}e_{i}(t) & i \in \mathscr{L} \\ \frac{1}{\left|N_{i}\right|} \sum_{j \in N_{i}}\left(y_{i}(t)-y_{j}(t)\right) & i \notin \mathscr{L} .\end{cases}
$$

$K_{1 i}, K_{2 i}, G_{1}$, and $G_{2}$ are designed matrices with appropriate dimensions.

(II) Distributed dynamic output feedback controller:

$$
\begin{gathered}
\dot{z}_{i}(t)=\bar{G}_{1 i} z_{i}(t)+\bar{G}_{2 i} e_{i \omega}(t), \\
u_{i}(t)=K_{i} z_{i}(t), \\
z_{i}(t) \in \mathbb{R}^{c+p_{i}}, \quad q \leq c, \quad i=1, \ldots, N .
\end{gathered}
$$

$K_{i}, \bar{G}_{1 i}$, and $\bar{G}_{2 i}$ are designed matrices with appropriate dimensions.

The variables $e_{i \omega}(t)$ in distributed feedback control laws (4) and (6) are measurable relative outputs. For $i \in \mathscr{L}, e_{i \omega}(t)$ is the regulated output $e_{i}(t)$. For $i \notin \mathscr{L}, e_{i \omega}(t)$ is the average of relative output errors between $i$ th agent and its neighbors.

In the sequel, we rewrite the closed-loop system into a composite form. Let $1_{N}$ be a column vector with all the elements as $1 . L_{i}$ and $l_{i}$, respectively, denote the $i$ th row of $L$ and $L 1_{N}$. For $i \in \mathscr{L}, l_{i}=1$, while for $i \notin \mathscr{L}, l_{i}=0$. Let $\chi=\left(x_{1}^{T}, z_{1}^{T}, \ldots, x_{N}^{T}, z_{N}^{T}\right)^{T}, e=\left(e_{1}^{T}, \ldots, e_{N}^{T}\right)^{T}$,

$$
\begin{aligned}
& \mathscr{A}_{1}=\operatorname{diag} \operatorname{block}\left(\mathscr{A}_{11}, \ldots, \mathscr{A}_{1 N}\right) \text {, } \\
& \mathscr{A}_{2}=\operatorname{diag} \operatorname{block}\left(\mathscr{A}_{21}, \ldots, \mathscr{A}_{2 \mathrm{~N}}\right) \text {, } \\
& \mathscr{C}_{1}=\operatorname{diag} \operatorname{block}\left(\left(\bar{C}_{1} 0_{n \times c}\right), \ldots,\left(\bar{C}_{N} 0_{n \times c}\right)\right) \text {, } \\
& \mathscr{C}_{2}=\operatorname{diag} \operatorname{block}\left(\left(\bar{C}_{1} 0_{n \times\left(c+p_{1}\right)}\right), \ldots,\left(\bar{C}_{N} 0_{n \times\left(c+p_{N}\right)}\right)\right) \text {, } \\
& \mathscr{G}_{1}=\left(\begin{array}{c}
L_{1} \otimes G_{21}^{\prime} \\
\vdots \\
L_{N} \otimes G_{2 N}^{\prime}
\end{array}\right), \quad \mathscr{G}_{2}=\left(\begin{array}{c}
L_{1} \otimes \bar{G}_{21}^{\prime} \\
\vdots \\
L_{N} \otimes \bar{G}_{2 N}^{\prime}
\end{array}\right), \\
& \mathscr{E}_{1}=\left(\begin{array}{c}
\bar{E}_{1} \\
l_{1} G_{2} F \\
\vdots \\
\bar{E}_{N} \\
l_{N} G_{2} F
\end{array}\right), \quad \mathscr{E}_{2}=\left(\begin{array}{c}
\bar{E}_{1} \\
l_{1} \bar{G}_{21} F \\
\vdots \\
\bar{E}_{N} \\
l_{N} \bar{G}_{2 N} F
\end{array}\right) \text {, }
\end{aligned}
$$

where $\mathscr{A}_{1 i}=\left(\begin{array}{cc}\bar{A}_{i}+\bar{B}_{i} K_{1 i} & \bar{B}_{i} K_{2 i} \\ 0 & G_{1}\end{array}\right), \mathscr{A}_{2 i}=\left(\begin{array}{cc}\bar{A}_{i} & \bar{B}_{i} K_{i} \\ 0 & \bar{G}_{1 i}\end{array}\right), G_{2 i}^{\prime}=\left(\begin{array}{c}0_{n \times p_{i}} \\ G_{2}\end{array}\right)$, and $\bar{G}_{2 i}^{\prime}=\left(\begin{array}{c}0_{n \times p_{i}} \\ \bar{G}_{2 i}\end{array}\right)$, for $i=1, \ldots, N$. Then, the closed-loop system (1), (2) with controllers (4) or (6) can be rewritten as

$$
\begin{gathered}
\dot{\omega}(t)=S \omega(t), \\
\dot{\chi}(t)=A_{c} \chi(t)+B_{c} \omega(t), \\
e(t)=C_{c} \chi(t)+F_{c} \omega(t) .
\end{gathered}
$$

With the controller (4),

$$
\begin{gathered}
A_{c}=\mathscr{A}_{1}+\mathscr{G}_{1} \mathscr{C}_{1}, \quad B_{c}=\mathscr{E}_{1}, \\
C_{c}=\mathscr{C}_{1}, \quad F_{c}=1_{N} \otimes F,
\end{gathered}
$$

while with the controller (6),

$$
\begin{gathered}
A_{c}=\mathscr{A}_{2}+\mathscr{G}_{2} \mathscr{C}_{2}, \quad B_{c}=\mathscr{E}_{2}, \\
C_{c}=\mathscr{C}_{2}, \quad F_{c}=1_{N} \otimes F .
\end{gathered}
$$

As a result, the distributed output regulation problem studied in this paper is given as follows.

Problem 3. Distributed output regulation problem: design controllers in the form of (4) or (6) such that the closed-loop system (8) has the following properties.

(i) It is exponentially stable at the origin with $\omega=0$.

(ii) For all initial values $\chi(0)$ and $\omega(0), \lim _{t \rightarrow \infty} e(t)=0$.

\section{Main Results}

In this section, we give two theorems about the solvability of Problem 3 and the approach of controller design. First of all, we introduce the concept of quadratic stability and related lemmas, which will be used later.

Definition 4 (Amato [28]). Consider a parametric uncertain linear system given by

$$
\dot{x}(t)=A\left(p_{0}\right) x(t), \quad t \in[0,+\infty),
$$

where $x(t) \in \mathbb{R}^{n_{0}}, p_{0} \in H \subset \mathbb{R}^{m_{0}}$ is the vector of uncertain parameters, where $H$ is a hyperbox, and $A(\cdot)$ is continuous. This system is said to be quadratically stable (QS) in $H$ if and only if there exists a symmetric positive definite matrix $P \in$ $\mathbb{R}^{n_{0} \times n_{0}}$ such that for all $p_{0} \in H, A^{T}\left(p_{0}\right) P+P A\left(p_{0}\right)<0$.

Lemma 5 (Amato [28]). Assume that the system (11) is QS. Then, for any function $p_{0}(\cdot)$ that is piecewise continuous on $\mathbb{R}^{+}$and valued on $W$, the linear time-varying system $\dot{x}(t)=$ $A\left(p_{0}(t)\right) x(t)$ is exponentially stable.

Lemma 6 (Amato [28]). Assume that the uncertain system is in a polytopic form; that is,

$$
\dot{x}(t)=A(t) x(t), \quad A(t) \in \operatorname{conv}\left\{A_{1}, A_{2}, \ldots, A_{g}\right\},
$$

where $A_{1}, \ldots, A_{g} \in \mathbb{R}^{n_{0} \times n_{0}}$ and conv $\{\cdot\}$ represents the convex hull of the following matrices. It is QS if and only if there exists a positive definite symmetric matrix $P$ such that for $k=$ $1, \ldots, g, A_{k}^{T} P+P A_{k}<0$. 
Second, we need to recall the concept of internal model and its property.

Definition 7 (Huang [23]). Given any square matrix $S$, a pair of matrices $\left(\mathscr{G}_{1}, \mathscr{G}_{2}\right)$ is said to incorporate a $p$-copy internal model of the matrix $S$ if the pair satisfies that

$$
\mathscr{G}_{1}=T\left(\begin{array}{cc}
S_{1} & S_{2} \\
0 & G_{1}
\end{array}\right) T^{-1}, \quad \mathscr{G}_{2}=T\left(\begin{array}{c}
S_{3} \\
G_{2}
\end{array}\right),
$$

where $S_{1}, S_{2}$, and $S_{3}$ are arbitrary constant matrices of appropriate dimensions, $T$ is any nonsingular matrix with the same dimension as $\mathscr{G}_{1}$, and $G_{1}, G_{2}$ are described as follows:

$$
\begin{aligned}
& G_{1}=\text { block diag }\left(\beta_{1}, \ldots, \beta_{p}\right), \\
& G_{2}=\text { block diag }\left(\sigma_{1}, \ldots, \sigma_{p}\right),
\end{aligned}
$$

where for $j=1, \ldots, p, \beta_{j}$ is a constant square matrix of dimension $d_{j}$ for some integer $d_{j}$ and $\sigma_{j}$ is a constant column vector of dimension $d_{j}$ such that

(i) $\beta_{i}$ and $\sigma_{i}$ are controllable,

(ii) the minimal polynomial of $S$ divides the characteristic polynomial of $\beta_{i}$.

Lemma 8 (Huang [23]). Under Assumption 1, assume that $\left(\mathscr{G}_{1}, \mathscr{G}_{2}\right)$ incorporates a $p$-copy internal model of $S$. Let

$$
\left[\begin{array}{cc}
\widehat{A} & \widehat{B} \\
\mathscr{G}_{2} \widehat{C} & \mathscr{G}_{1}
\end{array}\right]
$$

be exponentially stable, where $\widehat{A}, \widehat{B}$, and $\widehat{C}$ are any matrices with appropriate dimensions. Then, for any matrices $\widehat{E}$ and $\widehat{F}$ of appropriate dimensions, the following matrix equations

$$
\begin{gathered}
X S=\widehat{A} X+\widehat{B} Z+\widehat{E}, \\
Z S=\mathscr{G}_{1} Z+\mathscr{G}_{2}(\widehat{C} X+\widehat{F})
\end{gathered}
$$

have anique solution $X$ and $Z$. Moreover, $X$ satisfies $\widehat{C} X+\widehat{F}=$ 0 .

Based on these preparations, theorems on solvability of Problem 3 with state/output feedback controllers are given as follows.

Theorem 9. Suppose that Assumptions 1 and 2 hold and the pair $\left(G_{1}, G_{2}\right)$ is a p-copy internal model of the exosystem (1). For $i=1, \ldots, N$, if there exist a matrix $Y_{i}$ and a positive definite matrix $\widetilde{P}_{i}$ satisfying that

$$
\widetilde{A}_{k i} \widetilde{P}_{i}+\widetilde{P}_{i} \widetilde{A}_{k i}^{T}+\widetilde{B}_{k i} Y_{i}+Y_{i}^{T} \widetilde{B}_{k i}^{T}<0, \quad k=1, \ldots, g_{i},
$$

where

$$
\widetilde{A}_{k i}=\left(\begin{array}{cc}
A_{k i} & 0 \\
G_{2} C_{k i} & G_{1}
\end{array}\right), \quad \widetilde{B}_{k i}=\left(\begin{array}{c}
B_{k i} \\
0
\end{array}\right),
$$

Then, Problem 3 is solvable by a dynamic state feedback controller (4), where $\left(K_{1 i}, K_{2 i}\right)=K_{i}=Y_{i} \widetilde{P}_{i}^{-1}$.
Proof. Let $\gamma_{i}(t)=\left(x_{i}^{T}, z_{i}^{T}\right)^{T}$. Then, the subsystems of (8) with controller (4) can be written as follows: for $i \in \mathscr{L}$,

$$
\dot{\gamma}_{i}(t)=\left(\begin{array}{cc}
\bar{A}_{i}+\bar{B}_{i} K_{1 i} & \bar{B}_{i} K_{2 i} \\
G_{2} \bar{C}_{i} & G_{1}
\end{array}\right) \gamma_{i}(t)+\left(\begin{array}{c}
\bar{E}_{i} \\
G_{2} F
\end{array}\right) w(t),
$$

and for $i \notin \mathscr{L}$,

$$
\begin{aligned}
\dot{\gamma}_{i}(t)= & \left(\begin{array}{cc}
\bar{A}_{i}+\bar{B}_{i} K_{1 i} & \bar{B}_{i} K_{2 i} \\
G_{2} \bar{C}_{i} & G_{1}
\end{array}\right) \gamma_{i}(t)+\left(\begin{array}{c}
\bar{E}_{i} \\
0
\end{array}\right) w(t) \\
& +\frac{1}{\left|N_{i}\right|} \sum_{j \in N_{i}}\left(\begin{array}{cc}
0 & 0 \\
-G_{2} \bar{C}_{j} & 0
\end{array}\right) \gamma_{j}(t) .
\end{aligned}
$$

According to [29], by relabeling the nodes, a directed acyclic graph could be put into an ordered form. That is to say, for all edges $(i, j) \in \mathscr{E}, i>j$ holds. Notice that matrix $A_{c}$ is consequently a block lower triangular matrix and system (8) with $w=0$ is asymptotically stable if and only if all the subsystems below are asymptotically stable

$$
\begin{aligned}
\dot{\gamma}_{i}(t) & =\left(\begin{array}{cc}
\bar{A}_{i}+\bar{B}_{i} K_{1 i} & \bar{B}_{i} K_{2 i} \\
G_{2} \bar{C}_{i} & G_{1}
\end{array}\right) \gamma_{i}(t) \\
& =\sum_{k=1}^{g_{i}} \lambda_{k i}\left(\begin{array}{cc}
A_{k i}+B_{k i} K_{1 i} & B_{k i} K_{2 i} \\
G_{2} C_{k i} & G_{1}
\end{array}\right) \gamma_{i}(t) \\
& =\sum_{k=1}^{g_{i}} \lambda_{k i}\left(\widetilde{A}_{k i}+\widetilde{B}_{k i} K_{i}\right) \gamma_{i}(t) .
\end{aligned}
$$

Consider LMI (17). When it holds for a symmetric positive definite matrix $\widetilde{P}_{i}$ and a matrix $Y_{i}$, let $P_{i}=\widetilde{P}_{i}^{-1}$, and let $K_{i}=$ $Y_{i} P_{i}$. Pre- and postmultiplied by $P_{i},(17)$ is equivalent to the following inequality:

$$
\left(\widetilde{A}_{k i}+\widetilde{B}_{k i} K_{i}\right)^{T} P_{i}+P_{i}\left(\widetilde{A}_{k i}+\widetilde{B}_{k i} K_{i}\right)<0
$$

According to Lemma 6, when it holds, the subsystems (21) are all QS. And according to Lemma 5, for any polytopic uncertainties, the subsystems are exponentially stable. The system (8) with $w=0$ is consequently exponentially stable. The condition (i) of Problem 3 has been satisfied. In the following, the error $e(t)$ is proved to converge to zero.

Since the matrix $\left(\begin{array}{cc}\bar{A}_{i}+\bar{B}_{i} K_{1 i} & \bar{B}_{i} K_{2 i} \\ G_{2} \bar{C}_{i} & G_{1}\end{array}\right)$ is Hurwitz, according to Lemma 8 , for any $\bar{E}_{i}$ and $F_{i}$, the following matrix equations

$$
\begin{gathered}
X_{i} S=\left(\bar{A}_{i}+\bar{B}_{i} K_{1 i}\right) X_{i}+\bar{B}_{i} K_{2 i} Z_{i}+\bar{E}_{i} \\
Z_{i} S=G_{1} Z_{i}+G_{2}\left(\bar{C}_{i} X_{i}+F_{i}\right)
\end{gathered}
$$

have a unique solution $X_{i}$ and $Z_{i}$, and at the same time, $\bar{C}_{i} X_{i}+F_{i}=0$. For $i \in \mathscr{L}, F_{i}=F$, otherwise, $F_{i}=$ $\left(-1 /\left|N_{i}\right|\right) \sum_{j \in N_{i}} \bar{C}_{j} X_{j}$. Therefore, the $N$ coupled matrix equations have a unique solution $\left(X_{1}, Z_{1}, \ldots, X_{N}, Z_{N}\right)$.

The proof will be given by induction. As mentioned earlier, each agent can only receive information from the agents with smaller labels, after appropriately relabeling the 
directed acyclic graph. That is to say, for $i=1,2, \ldots, N, F_{i}=F$ or $F_{i}=\left(-1 /\left|N_{i}\right|\right) \sum_{j=1, j \in N_{i}}^{i-1} \bar{C}_{j} X_{j}$. For $i=1$, the first agent does not communicate with any other agents but the exosystem, so $F_{1}=F$. And we can obtain a unique pair of $\left(X_{1}, Z_{1}\right)$ that satisfies (23) and $\bar{C}_{1} X_{1}+F_{1}=0$. For $i=2$, if it is a leader, then $F_{2}=F$; or else, it can only communicate with the first agent, which means that $F_{2}=-\bar{C}_{1} X_{1}$. In both cases $F_{2}$ is a certain and known matrix, so there exists a unique solution $\left(X_{2}, Z_{2}\right)$ of (23) and $\bar{C}_{2} X_{2}+F_{2}=0$. Suppose that for $i=1,2, \ldots, m$, the solution $\left(X_{i}, Z_{i}\right)$ has already been obtained. And then for $i=m+1$, either $F_{i}=F$ or $F_{i}=$ $\left(-1 /\left|N_{i}\right|\right) \sum_{j=1, j \in N_{i}}^{i-1} \bar{C}_{j} X_{j}$ is a certain and known matrix. So, the unique solution $\left(X_{m+1}, Z_{m+1}\right)$ also exists. By induction, we can obtain the unique solution $\left(X_{1}, Z_{1}, \ldots, X_{N}, Z_{N}\right)$, and at the same time, $\bar{C}_{1} X_{1}+F_{1}=\cdots=\bar{C}_{N} X_{N}+F_{N}=0$.

Take notations $\Gamma_{i}=\left(\begin{array}{c}X_{i} \\ Z_{i}\end{array}\right)$ and $\widetilde{\gamma}_{i}=\gamma_{i}-\Gamma_{i} \omega$, and substitute (23) into (19) and (20). It is obtained that

$$
\begin{aligned}
\dot{\tilde{\gamma}}_{i}(t) & =\dot{\gamma}_{i}(t)-\Gamma_{i} S \omega(t) \\
= & \left(\begin{array}{cc}
\bar{A}_{i}+\bar{B}_{i} K_{1 i} & \bar{B}_{i} K_{2 i} \\
G_{2} \bar{C}_{i} & G_{1}
\end{array}\right) \tilde{\gamma}_{i}(t), \quad i \in \mathscr{L}, \\
\dot{\tilde{\gamma}}_{i}(t)= & \dot{\gamma}_{i}(t)-\Gamma_{i} S \omega(t) \\
= & \left(\begin{array}{cc}
\bar{A}_{i}+\bar{B}_{i} K_{1 i} & \bar{B}_{i} K_{2 i} \\
G_{2} \bar{C}_{i} & G_{1}
\end{array}\right) \tilde{\gamma}_{i}(t) \\
& -\frac{1}{\left|N_{i}\right|} \sum_{j \in N_{i}}\left(\begin{array}{cc}
0 & 0 \\
G_{2} \bar{C}_{j} & 0
\end{array}\right) \widetilde{\gamma}_{j}(t), \quad i \notin \mathscr{L} .
\end{aligned}
$$

By the first part of the proof, the system above is exponentially stable; that is,

$$
\lim _{t \rightarrow \infty} \tilde{\gamma}_{i}(t)=0, \quad i=1, \ldots, N
$$

Since

$$
\begin{aligned}
e_{i w}(t)= & \bar{C}_{i} x_{i}(t)+F \omega(t) \\
= & \left(\bar{C}_{i} 0\right) \widetilde{\gamma}_{i}(t)+\left(\bar{C}_{i} X_{i}+F\right) \omega(t), \quad i \in \mathscr{L}, \\
e_{i \omega}(t)= & \bar{C}_{i} x_{i}(t)-\frac{1}{\left|N_{i}\right|} \sum_{j \in N_{i}} \bar{C}_{j} x_{j}(t) \\
= & \left(\bar{C}_{i} 0\right) \widetilde{\gamma}_{i}(t)-\frac{1}{\left|N_{i}\right|} \sum_{j \in N_{i}}\left(\bar{C}_{j} 0\right) \widetilde{\gamma}_{j}(t) \\
& +\left(\bar{C}_{i} X_{i}+F_{i}\right) \omega(t), \quad i \notin \mathscr{L},
\end{aligned}
$$

it is obvious that

$$
\lim _{t \rightarrow \infty} e_{i \omega}(t)=0, \quad i=1, \ldots, N .
$$

Under Assumption 2, the statement (27) is equivalent to $\lim _{t \rightarrow \infty} e(t)=0$. This completes the proof.

Remark 10. When there is no uncertainty in the system (2), $\bar{A}_{i}=A_{1 i}, \bar{B}_{i}=B_{1 i}$, and $\bar{C}_{i}=C_{1 i}, i=1, \ldots, N$. Then, the conclusion of Theorem 9 still holds if the solvability of LMI (17) is replaced by the statement that the pair $\left(A_{1 i}, B_{1 i}\right)$ is stabilizable. In fact, according to [23], from the two statements,

(i) the pair $\left(A_{1 i}, B_{1 i}\right)$ is stabilizable,

(ii) Assumption 1 holds, and the pair $\left(G_{1}, G_{2}\right)$ is an internal model of $S$; it is followed that the pair

$$
\left(\left(\begin{array}{cc}
A_{1 i} & 0 \\
G_{2} C_{1 i} & G_{1}
\end{array}\right),\left(\begin{array}{c}
B_{1 i} \\
0
\end{array}\right)\right)
$$

is stabilizable. It is equivalent to LMI (17) that holds with $g_{i}=$ $1, i=1, \ldots, N$. Specially, if for $i=1, \ldots, N, A_{1 i}=A, B_{2 i}=B$, and $C_{1 i}=C, g_{i}=1$, then in Theorem 9 , the solvability of LMI (17) can be replaced by the statement that the pair $(A, B)$ is stabilizable.

Theorem 11. Suppose that Assumptions 1 and 2 hold and the pair $\left(G_{1}, G_{2}\right)$ is an internal model of the exosystem (1) if the following conditions are satisfied:

(i) For $i=1, \ldots, N$, there exist a matrix $Y_{i}$ and $a$ symmetric positive definite matrix $\widetilde{P}_{i}$ satisfying LMI (17),

(ii) For $i=1, \ldots, N$, and the matrices $Y_{i}, \widetilde{P}_{i}$ obtained in (i), let $P_{i}=\widetilde{P}_{i}^{-1}$, and let $K_{i}=\left(K_{1 i}, K_{2 i}\right)=Y_{i} P_{i}$, and there exist matrices $M_{1 i}, M_{2 i}, M_{3 i}$ and a symmetric positive definite matrix $Q_{i}$ satisfying the following $L M I$ :

$$
\left(\begin{array}{ll}
\bar{\Pi}_{11, k i} & \bar{\Pi}_{12, k i} \\
\bar{\Pi}_{12, k i}^{T} & \bar{\Pi}_{22, k i}
\end{array}\right)<0,
$$

where

$$
\begin{gathered}
\bar{\Pi}_{11, k i}=P_{i} \widetilde{A}_{k i}+\widetilde{A}_{k i}^{T} P_{i}+\widetilde{B}_{k i} K_{i} P_{i}+P_{i} K_{i}^{T} \widetilde{B}_{k i}^{T}, \\
\bar{\Pi}_{12, k i}=P_{i}\left(\begin{array}{c}
B_{k i} K_{1 i} \\
0
\end{array}\right) \\
+\left(\begin{array}{c}
-A_{k i}^{T} Q_{i}-K_{1 i}^{T} B_{k i} Q_{i}+M_{1 i}^{T}+C_{k i}^{T} M_{3 i}^{T} \\
-K_{2 i}^{T} B_{k i}^{T} Q_{i}+M_{2 i}^{T}
\end{array}\right), \\
\bar{\Pi}_{22, k i}=-Q_{i} B_{k i} K_{1 i}-K_{1 i}^{T} B_{k i}^{T} Q_{i}+M_{1 i}+M_{1 i}^{T}, \\
k=1, \ldots, g_{i} .
\end{gathered}
$$

Then, Problem 3 is solvable by a dynamic output feedback controller (6) with

$$
\bar{G}_{1 i}=\left(\begin{array}{cc}
T_{1 i} & T_{2 i} \\
0 & G_{1}
\end{array}\right), \quad \bar{G}_{2 i}=\left(\begin{array}{c}
T_{3 i} \\
G_{2}
\end{array}\right),
$$

where $T_{1 i}=Q_{i}^{-1} M_{1 i}, T_{2 i}=Q_{i}^{-1} M_{2 i}$, and $T_{3 i}=Q_{i}^{-1} M_{3 i}$.

Proof. For $i=1, \ldots, N$, the subsystems of (8) with (6) and (31) can be written in such a form: for $i \in \mathscr{L}$,

$$
\dot{\gamma}_{i}(t)=\left(\begin{array}{ccc}
\bar{A}_{i} & \bar{B}_{i} K_{1 i} & \bar{B}_{i} K_{2 i} \\
T_{3 i} \bar{C}_{i} & T_{1 i} & T_{2 i} \\
G_{2} \bar{C}_{i} & 0 & G_{1}
\end{array}\right) \gamma_{i}(t)+\left(\begin{array}{c}
\bar{E}_{i} \\
T_{3 i} F \\
G_{2} F
\end{array}\right) w(t),
$$


and for $i \notin \mathscr{L}$,

$$
\begin{aligned}
\dot{\gamma}_{i}(t)= & \left(\begin{array}{ccc}
\bar{A}_{i} & \bar{B}_{i} K_{1 i} & \bar{B}_{i} K_{2 i} \\
T_{3 i} \bar{C}_{i} & T_{1 i} & T_{2 i} \\
G_{2} \bar{C}_{i} & 0 & G_{1}
\end{array}\right) \gamma_{i}(t)+\left(\begin{array}{c}
\bar{E}_{i} \\
0 \\
0
\end{array}\right) w(t) \\
& -\frac{1}{\left|N_{i}\right|} \sum_{j \in N_{i}}\left(\begin{array}{ccc}
0 & 0 & 0 \\
T_{3 i} \bar{C}_{j} & 0 & 0 \\
G_{2} \bar{C}_{j} & 0 & 0
\end{array}\right) \gamma_{j}(t) .
\end{aligned}
$$

Similar to the proof of the previous theorem, the stability of the system (8) with $\omega=0$ is dependent on the following subsystems:

$$
\begin{aligned}
\dot{\gamma}_{i}(t) & =\left(\begin{array}{ccc}
\bar{A}_{i} & \bar{B}_{i} K_{1 i} & \bar{B}_{i} K_{2 i} \\
T_{3 i} \bar{C}_{i} & T_{1 i} & T_{2 i} \\
G_{2} \bar{C}_{i} & 0 & G_{1}
\end{array}\right) \gamma_{i}(t) \\
& =\sum_{k=1}^{g_{i}} \lambda_{k i}\left(\begin{array}{ccc}
A_{k i} & B_{k i} K_{1 i} & B_{k i} K_{2 i} \\
T_{3 i} C_{k i} & T_{1 i} & T_{2 i} \\
G_{2} C_{k i} & 0 & G_{1}
\end{array}\right) \gamma_{i}(t) .
\end{aligned}
$$

Let

$$
\xi_{i}(t)=\left(\begin{array}{ccc}
I_{p_{i}} & 0 & 0 \\
0 & 0 & I_{c} \\
-I_{p_{i}} & I_{p_{i}} & 0
\end{array}\right) \gamma_{i}(t)
$$

Then, the subsystem (34) is similar to the following system:

$$
\dot{\xi}_{i}(t)=\sum_{k=1}^{g_{i}} \lambda_{k i}\left(\begin{array}{ccc}
A_{k i}+B_{k i} K_{1 i} & B_{k i} K_{2 i} & B_{k i} K_{1 i} \\
G_{2} C_{k i} & G_{1} & 0 \\
U_{k i} & V_{k i} & W_{k i}
\end{array}\right) \xi_{i}(t),
$$

where

$$
\begin{gathered}
U_{k i}=T_{1 i}+T_{3 i} C_{k i}-A_{k i}-B_{k i} K_{1 i}, \\
V_{k i}=T_{2 i}-B_{k i} K_{2 i}, \quad W_{k i}=T_{1 i}-B_{k i} K_{1 i} .
\end{gathered}
$$

According to Lemma 6, the system (36) is QS if there exists a symmetric positive definite matrix $R_{i}>0$ such that

$$
\begin{aligned}
& \left(\begin{array}{ccc}
A_{k i}+B_{k i} K_{1 i} & B_{k i} K_{2 i} & B_{k i} K_{1 i} \\
G_{2} C_{k i} & G_{1} & 0 \\
U_{k i} & V_{k i} & W_{k i}
\end{array}\right)^{T} R_{i} \\
& \quad+R_{i}\left(\begin{array}{ccc}
A_{k i}+B_{k i} K_{1 i} & B_{k i} K_{2 i} & B_{k i} K_{1 i} \\
G_{2} C_{k i} & G_{1} & 0 \\
U_{k i} & V_{k i} & W_{k i}
\end{array}\right)<0 .
\end{aligned}
$$

Suppose that $R_{i}$ is a block diagonal matrix, which means that $R_{i}=\left(\begin{array}{cc}P_{i} & 0 \\ 0 & Q_{i}\end{array}\right), P_{i} \in \mathbb{R}^{\left(p_{i}+c\right) \times\left(p_{i}+c\right)}$, and $Q_{i} \in \mathbb{R}^{p_{i} \times p_{i}}$. Then, the inequality above can be rewritten as

$$
\left(\begin{array}{ll}
\Pi_{11, k i} & \Pi_{12, k i} \\
\Pi_{12, k i}^{T} & \Pi_{22, k i}
\end{array}\right)<0
$$

where

$$
\begin{gathered}
\Pi_{11, k i}=\left(\widetilde{A}_{k i}+\widetilde{B}_{k i} K_{i}\right)^{T} P_{i}+P_{i}\left(\widetilde{A}_{k i}+\widetilde{B}_{k i} K_{i}\right), \\
\Pi_{12, k i}=P_{i}\left(\begin{array}{c}
B_{k i} K_{1 i} \\
0
\end{array}\right)+\left(\begin{array}{c}
U_{k i}^{T} \\
V_{k i}^{T}
\end{array}\right) Q_{i}, \\
\Pi_{22, k i}=W_{k i}^{T} Q_{i}+Q_{i} W_{k i} .
\end{gathered}
$$

Notice that $\Pi_{11, k i}<0$ is just the inequality (22), which is equivalent to LMI (17). When $\Pi_{11, k i}<0$ holds, the matrices $\widetilde{P}_{i}, Y_{i}, P_{i}=\widetilde{P}_{i}^{-1}, K_{i}=\left(K_{1 i}, K_{2 i}\right)=Y_{i} \widetilde{P}_{i}$ have already been obtained. Let $Q_{i} T_{1 i}=M_{1 i}, Q_{i} T_{2 i}=M_{2 i}$, and $Q_{i} T_{3 i}=M_{3 i}$. We turn the inequality (39) into LMI (29). This implies that the system (8) with $\omega=0$ is asymptotically stable.

The rest part of the proof is similar to that in Theorem 9. Recalling that $\left(\begin{array}{cc}\bar{A}_{i} & \bar{B}_{i} K_{i} \\ \bar{G}_{2 i} \bar{C}_{i} & \bar{G}_{1 i}\end{array}\right)$ is Hurwitz and $\left(\bar{G}_{1 i}, \bar{G}_{2 i}\right)$ incorporates a $p$-copy internal model of $S$, we can obtain that for any matrices $\bar{E}_{i}$ and $F_{i}$, the following matrix equations

$$
\begin{gathered}
\bar{X}_{i} S=\bar{A}_{i} \bar{X}_{i}+\bar{B}_{i} K_{i} \bar{Z}_{i}+\bar{E}_{i}, \\
\bar{Z}_{i} S=\bar{G}_{1 i} \bar{Z}_{i}+\bar{G}_{2 i}\left(\bar{C}_{i} \bar{X}_{i}+F_{i}\right)
\end{gathered}
$$

have a unique solution $\bar{X}_{i}$ and $\bar{Z}_{i}$. And meanwhile, $\bar{C}_{i} \bar{X}_{i}+F_{i}=$ 0 . For $i \in \mathscr{L}, F_{i}=F$, otherwise $F_{i}=\left(-1 /\left|N_{i}\right|\right) \sum_{j \in N_{i}} \bar{C}_{j} \bar{X}_{j}$.

Take notations $\bar{\Gamma}_{i}=\left(\begin{array}{c}\bar{X}_{i} \\ \bar{Z}_{i}\end{array}\right)$ and $\bar{\gamma}_{i}(t)=\gamma_{i}(t)-\bar{\Gamma}_{i} \omega(t)$. By calculation, it can be verified that $\lim _{t \rightarrow \infty} \bar{\gamma}_{i}(t)=0$. Consequently, $\lim _{t \rightarrow \infty} e_{i \omega}(t)=0$. When Assumption 2 holds, the above statement is equivalent to $\lim _{t \rightarrow \infty} e(t)=0$. This completes the proof.

Remark 12. Consider the robust tracking problem for heterogenous MAS in the presence of disturbances. Suppose that an active leader is described as

$$
\dot{x}_{k}(t)=A_{k} x_{k}(t), \quad y_{k}(t)=C_{k} x_{k}(t),
$$

and there is an environmental disturbance $d(t)$ satisfying

$$
\dot{d}(t)=A_{d} d(t) .
$$

The model of the MAS can be given in

$$
\begin{gathered}
\dot{x}_{i}(t)=\bar{A}_{i} x_{i}(t)+\bar{B}_{i} u_{i}(t)+E_{d i} d(t), \\
y_{i}(t)=\bar{C}_{i} x_{i}(t), \\
i=1, \ldots, N,
\end{gathered}
$$

where $\bar{A}_{i}, \bar{B}_{i}, \bar{C}_{i}$, and $E_{d} i$ are uncertain matrices with polytopic uncertainties. The control target is to design distributed controllers such that the outputs of followers track the output of the leader. That is to say, for $i=1, \ldots, N, e_{i}(t)=y_{i}(t)-$ $y_{k}(t)$ converges to zero. This problem is just Problem 3 with $\omega=\left(x_{k}^{T}, d^{T}\right)^{T}, S=\operatorname{diag}$ block $\left(A_{k}, A_{d}\right), \bar{E}=\left(\begin{array}{ll}0 & E_{d i}\end{array}\right)$, and $F=\left(\begin{array}{ll}-C_{k} & 0\end{array}\right)$. The robust tracking problem for the systems (44), therefore, can be studied under the framework of DOR problem. It can be solved by designing the controller (4) or (6) if the conditions in Theorems 9 or 11 are satisfied. 
Remark 13. By Theorems 9 and 11, the sufficient condition of the solvability for Problem 3 depends on the dynamics of each agent. For a heterogenous MAS, $N$ LMIs have to be solved to obtain appropriate distributed controllers, which limits scalability. When the agents share some common characteristics, such as nominal parts, the complexity of LMIs will decrease. Specially, if agents are identical in the sense of common nominal parts and common bounds of the uncertainties, the solution to one LMI will construct the required control law.

\section{A Numerical Example}

In this section, a numerical example of a heterogenous MAS consisting seven agents is given to show the control effect of controllers obtained by Theorems 9 and 11. A comparison is also given between our controllers and that of [25]. In the example, suppose that there is a constant environmental noise and the reference signal (the output of the tracking target) is a sinusoidal signal. Let $\omega=\left(\omega_{1}, \omega_{2}, \omega_{3}\right)^{T}$, where $\omega_{1}, \omega_{2}$ serve as the reference signals and $\omega_{3}$ as the exogenous disturbance. Obviously, the differential equations about them are $\ddot{\omega}_{1}(t)=-\omega_{1}(t), \ddot{\omega}_{2}(t)=-\omega_{2}(t)$, and $\ddot{\omega}_{3}(t)=0$. Therefore, the exosystem can be described by (1) with

$$
S=\left(\begin{array}{ccc}
0 & 1 & 0 \\
-1 & 0 & 0 \\
0 & 0 & 0
\end{array}\right)
$$

According to [23], we can immediately obtain a 1-copy internal model as follows:

$$
G_{1}=\left(\begin{array}{ccc}
0 & 1 & 0 \\
0 & 0 & 1 \\
0 & -1 & 0
\end{array}\right), \quad G_{2}=\left(\begin{array}{l}
0 \\
0 \\
1
\end{array}\right) .
$$

In the example, three kinds of linear agents with 2, 3, and 4 orders are considered. 2 or 3 basement matrices are randomly valued for each kind. We present the detailed description of basement matrices in the appendix.

And as for $\bar{E}_{1}, \ldots, \bar{E}_{7}$, their first two columns are zeros, noticing that only $\omega_{3}$ refers to the disturbance, while the other elements are valued randomly. Finally, the regulated output, regarded as the tracking error between $y_{i}(t)$ and the sine reference signal $\omega_{1}(t)$, is defined as $e_{i}(t)=y_{i}(t)-\omega_{1}(t)=$ $\bar{C}_{i} x_{i}(t)+F \omega(t)$, so, value $F=\left(\begin{array}{lll}-1 & 0 & 0\end{array}\right)$.

The communication network is described by the directed acyclic graph shown in Figure 1, which satisfies Assumption 2. According to Theorem 11, by solving three kinds of LMIs as (17), we get control gains $K_{1}=K_{2}=$ $(-6.3230,28.5507,2.6636,0.9019,6.8977), K_{3}=K_{4}=$ $K_{5}=(0.9071,-9.9908,10.4217,-1.2503,0.9513,-3.1324)$, $K_{6}=K_{7}=(226.6539,117.7418,-20.0749,-91.1218,4.6064$, $-2.6648,11.6774)$. If the states of each agent are measurable, then a candidate distributed controller can be chosen as (4) with the coefficients $K_{1}, \ldots, K_{7}$. Or else, one more group of LMIs (29) need to be solved to construct the output feedback controllers. In this situation, we obtain $T_{1 i}, T_{2 i}$ and $T_{3 i}$ for $i=1, \ldots, 7$, which are also listed in the appendix due to

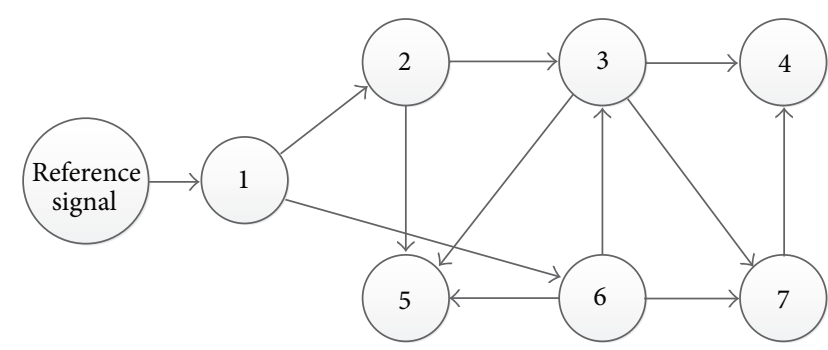

FIGURE 1: Information flow among exosystem and agents.

space limitation. And then the distributed dynamic output feedback controllers are given as (6). For simplicity, we denote this control law as controller $C_{a}$.

On the other hand, we translate the polytopic uncertainty into an equivalent form that consists of a nominal part and an uncertain part. In this case, the nominal part $A_{1}, \ldots, A_{7}$, $B_{1}, \ldots, B_{7}$, and $C_{1}, \ldots, C_{7}$ can be valued as the average of the basement matrices. Then, by the algorithm in [25], for $i=$ $1, \ldots, 7$, the candidate control law can be chosen as (6) with

$$
\begin{gathered}
\bar{G}_{1 i}=\left(\begin{array}{rr}
A_{i}+B_{i} K_{1 i}+L_{i} C_{i} & B_{i} K_{2 i} \\
0 & G_{1}
\end{array}\right), \\
\bar{G}_{2 i}=\left(\begin{array}{c}
-L_{i} \\
G_{2}
\end{array}\right), \quad J_{i}=Q_{i}^{-1} Z_{i},
\end{gathered}
$$

where $K_{1}=K_{2}=(-10,0,2,1,-1), L_{1}=L_{2}=(9.5,10)^{T}$, $K_{3}=K_{4}=K_{5}=(-9,-2,-4,3,2,4), L_{3}=L_{4}=L_{5}=$ $(30,-70,28)^{T}, K_{6}=K_{7}=(10,10,-2,5,1,2,-2)^{T}, L_{5}=L_{6}=$ $L_{7}=(-69,-41,-10,-10)^{T}$. For simplicity, this control law obtained by [25] is denoted by controller $C_{b}$.

Note that both controllers $C_{a}$ and $C_{b}$ are robust controllers against the model uncertainty. To compare the effects, two cases of uncertainties are considered in the example. For one case, the model uncertainties denoted by $\Delta_{1}$ are rather close to the "nominal part" set earlier. For $i=1, \ldots, 7$, $k=1, \ldots, g_{i}$, the values of $\lambda_{k i}$ are randomly chosen around $1 / g_{i}$, whose values are shown in the appendix. For another case, the model uncertainties denoted by $\Delta_{2}$ are valued freely with reasonable coefficients, which are relatively farther from the "nominal part." With the same initial values, the numerical results of the regulated output for each agent are demonstrated in Figure 2 and Figure 3. Our controller $C_{a}$ is effective in the presence of either $\Delta_{1}$ or $\Delta_{2}$. However, as for Controller $C_{b}$, when the uncertainty is relatively small, it can help to achieve the output regulation property. While in the case of $\Delta_{2}$, the regulated errors diverge rapidly as shown in Figure 3(b). As a consequence of the above, for Problem 3 with polytopic uncertainties, Theorems 9 and 11 help construct distributed dynamic state/output feedback controllers of stronger robustness, at the price of increasing the calculation complexity.

\section{Conclusions}

In this paper, we consider the distributed robust output regulation problem for MASs under the topology of directed 


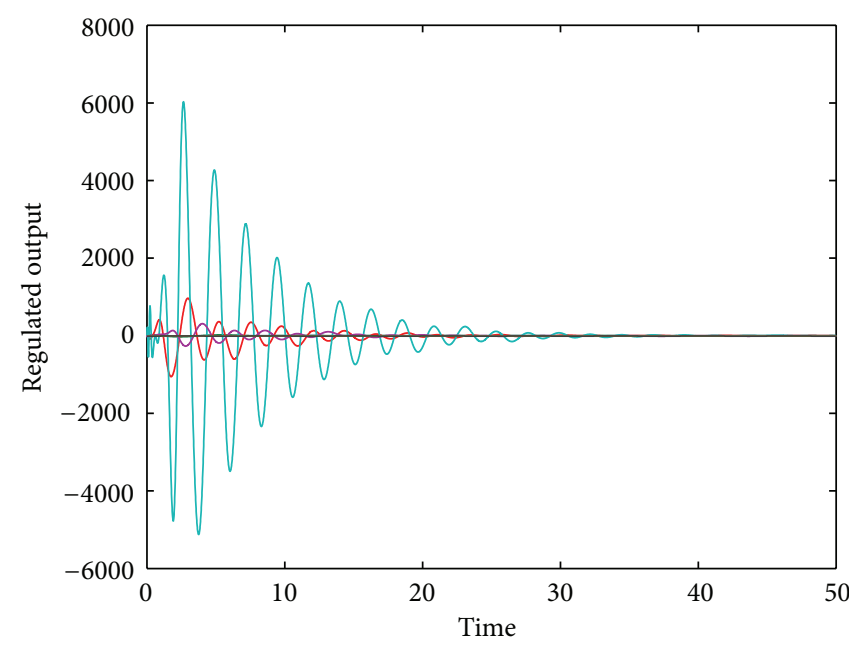

(a) For uncertainty $\Delta_{1}$

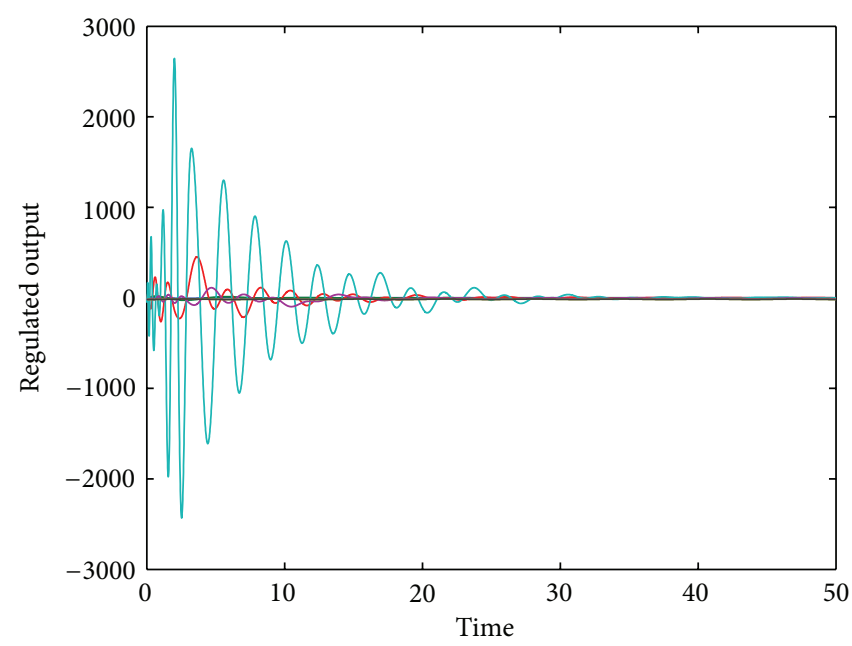

(b) For uncertainty $\Delta_{2}$

FIGURE 2: Regulated output of the MAS with the controller $C_{a}$.

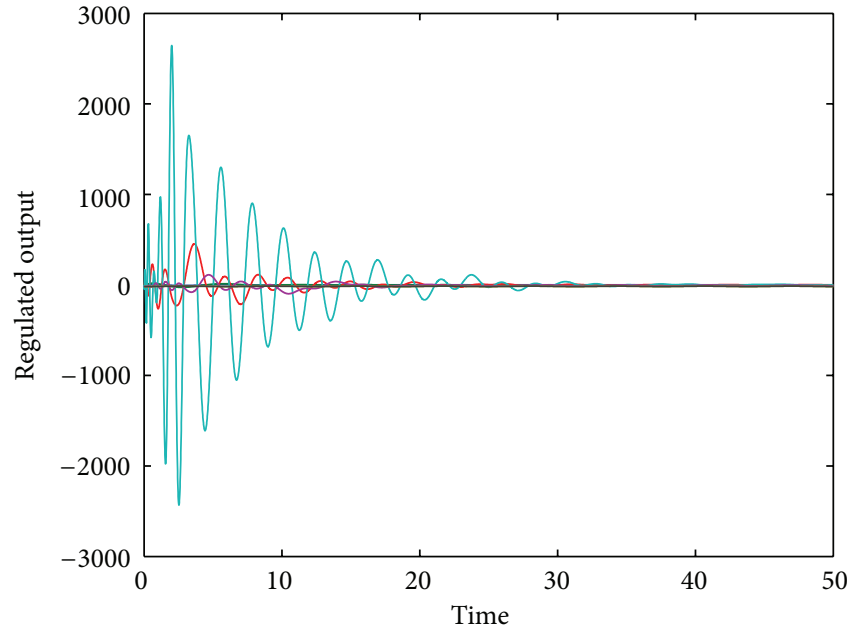

(a) For uncertainty $\Delta_{1}$

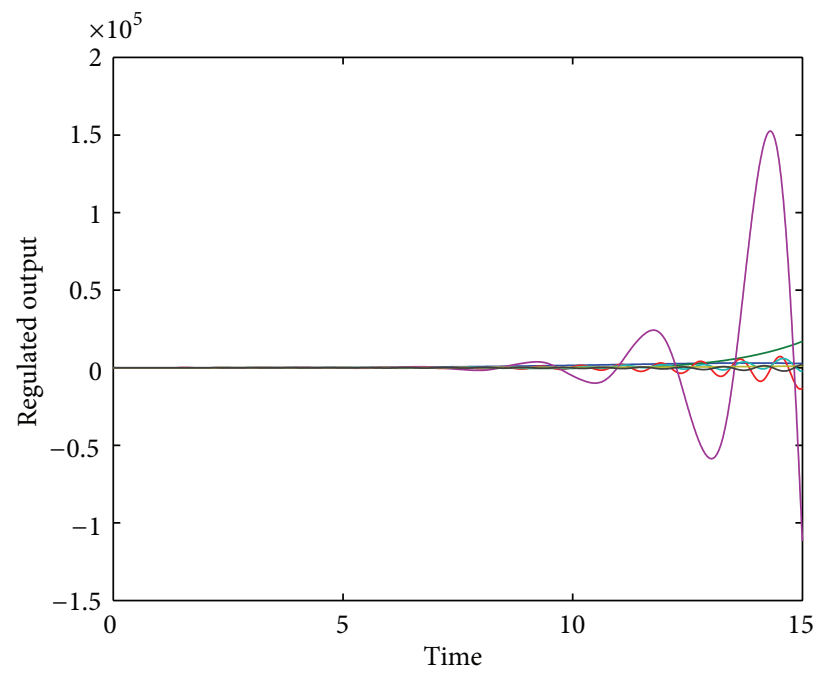

(b) For uncertainty $\Delta_{2}$

FIGURE 3: Regulated output of the MAS with the controller $C_{b}$.

acyclic graphs. As an extension of the existing results, we focus on a special class of parametric uncertainty. Assume that the dynamics of heterogeneous agents are in a polytopic form, and we study the solvability of the problem and the design of distributed controllers. Both dynamic state feedback controller and dynamic output feedback controller are under consideration. At last, a practical example is presented to validate our results. By local interaction, a team of heterogenous agents is required to achieve a common output, which is generated by an exosystem. The controller generated by our theorem can realize the property of output regulation and the performance of tracking the reference signal and rejecting the disturbances as a special case.

\section{Appendix}

Here are some detailed descriptions of the example in Section 4 . Agents $v_{1}$ and $v_{2}$ are systems with 2 orders. And there are three basement matrices for an uncertain system, which means $g_{1}=g_{2}=3$

$$
\begin{aligned}
& A_{11}=A_{12}=\left(\begin{array}{cc}
-4.8512 & -8.9578 \\
-8.7108 & 7.4003
\end{array}\right), \\
& A_{21}=A_{22}=\left(\begin{array}{cc}
-4.5841 & -7.5263 \\
-9.3135 & 7.0596
\end{array}\right), \\
& A_{31}=A_{32}=\left(\begin{array}{cc}
-5.7140 & -8.2750 \\
-9.1175 & 7.0544
\end{array}\right),
\end{aligned}
$$




$$
\begin{gathered}
B_{11}=B_{12}=\left(\begin{array}{c}
1.6684 \\
-0.0861
\end{array}\right), \\
B_{21}=B_{22}=\left(\begin{array}{c}
1.9897 \\
-0.7854
\end{array}\right), \quad B_{31}=B_{32}=\left(\begin{array}{c}
1.8509 \\
-0.2403
\end{array}\right), \\
C_{11}=C_{12}=\left(\begin{array}{ll}
1.0614 & 0.8390
\end{array}\right), \\
C_{21}=C_{22}=\left(\begin{array}{ll}
1.0102 & 0.9982
\end{array}\right), \\
C_{31}=C_{32}=\left(\begin{array}{ll}
0.9283 & 0.7936
\end{array}\right) .
\end{gathered}
$$

Agents $v_{3}, v_{4}$, and $v_{5}$ are systems with 3 orders. And $g_{3}=g_{4}=$ $g_{5}=2$

$$
\begin{aligned}
& A_{13}=A_{14}=A_{15}=\left(\begin{array}{ccc}
2.5419 & -1.2319 & 4.7038 \\
1.9598 & -1.9843 & -4.6719 \\
-2.6654 & -2.4159 & -4.6598
\end{array}\right) \text {, } \\
& A_{23}=A_{24}=A_{25}=\left(\begin{array}{ccc}
1.1025 & -2.1701 & 3.6488 \\
0.0927 & -0.1010 & -2.6299 \\
-3.4064 & -3.1707 & -3.2973
\end{array}\right) \text {, } \\
& B_{13}=B_{14}=B_{15}=\left(\begin{array}{c}
3.2738 \\
2.3681 \\
-0.0088
\end{array}\right) \text {, } \\
& B_{23}=B_{24}=B_{25}=\left(\begin{array}{c}
4.3570 \\
1.9363 \\
-0.0489
\end{array}\right) \text {, } \\
& C_{13}=C_{14}=C_{15}=\left(\begin{array}{lll}
-2.9090 & -1.6423 & -0.6208
\end{array}\right) \text {, } \\
& C_{23}=C_{24}=C_{25}=\left(\begin{array}{lll}
-3.5357 & -1.0246 & -2.3913
\end{array}\right) \text {. }
\end{aligned}
$$

Agents $v_{6}$ and $v_{7}$ are systems with 4 orders. And $g_{6}=g_{7}=3$

$$
\begin{aligned}
& A_{16}=A_{17}=\left(\begin{array}{cccc}
7.3858 & 7.0606 & -1.9638 & -6.3218 \\
1.5941 & 2.4411 & -8.4807 & -5.2009 \\
0.9972 & -2.9810 & -5.2017 & -1.6547 \\
-7.1009 & 0.2650 & -7.5336 & -9.0069
\end{array}\right), \\
& A_{26}=A_{27}=\left(\begin{array}{cccc}
5.7750 & 7.7097 & -3.0848 & -4.7077 \\
-0.1851 & 0.8409 & -8.0396 & -3.7288 \\
1.0337 & -2.4579 & -4.1684 & -3.4229 \\
-7.0579 & 1.8202 & -7.1493 & -10.8314
\end{array}\right), \\
& A_{36}=A_{37}=\left(\begin{array}{cccc}
7.6867 & 8.3454 & -1.3674 & -6.1338 \\
-0.1668 & 0.5027 & -7.5538 & -6.0157 \\
-0.0637 & -4.8089 & -4.6107 & -0.6759 \\
-7.6883 & -1.0590 & -7.7299 & -10.2511
\end{array}\right), \\
& B_{16}=B_{17}=\left(\begin{array}{c}
0.0681 \\
0.0862 \\
2.5410 \\
2.3587
\end{array}\right),
\end{aligned}
$$

$$
\begin{gathered}
B_{26}=B_{27}=\left(\begin{array}{c}
-0.2206 \\
0.3290 \\
1.9179 \\
2.2930
\end{array}\right), \\
B_{36}=B_{37}=\left(\begin{array}{c}
-0.2305 \\
0.9642 \\
3.2929 \\
2.4590
\end{array}\right), \\
C_{16}=C_{17}=\left(\begin{array}{lll}
1.4942 & -2.5319-1.0521 & 1.0049
\end{array}\right), \\
C_{26}=C_{27}=\left(\begin{array}{lll}
0.9337 & -1.6942-1.9109 & 0.4535
\end{array}\right), \\
C_{36}=C_{37}=\left(\begin{array}{lll}
1.4678 & -2.6602-1.1586 & 0.6176
\end{array}\right) .
\end{gathered}
$$

Next, these are the matrices obtained according to the solution of LMIs (29)

$$
\begin{aligned}
& T_{11}=T_{12}=\left(\begin{array}{cc}
37.5608 & 109.7053 \\
-118.3196 & -149.5498
\end{array}\right), \\
& T_{21}=T_{22}=\left(\begin{array}{ccc}
4.8984 & 1.6586 & 12.6849 \\
-1.7007 & -0.5759 & -4.4042
\end{array}\right) \text {, } \\
& T_{31}=T_{32}=\left(\begin{array}{cc}
-43.5409 & -74.3826 \\
91.9349 & 157.0558
\end{array}\right) \text {, } \\
& T_{13}=T_{14}=T_{15}=\left(\begin{array}{ccc}
-6.8197 & -42.1931 & 33.9132 \\
9.9034 & -14.7875 & 19.6377 \\
-2.6741 & -1.6089 & -3.5333
\end{array}\right) \text {, } \\
& T_{23}=T_{24}=T_{25}=\left(\begin{array}{ccc}
-4.2895 & 3.2637 & -10.7466 \\
-2.3038 & 1.7529 & -5.7718 \\
0.1139 & -0.0867 & 0.2854
\end{array}\right) \text {, } \\
& T_{33}=T_{34}=T_{35}=\left(\begin{array}{ccc}
11.1566 & 5.9291 & 5.2847 \\
-7.3616 & -3.9123 & -3.4871 \\
-0.3312 & -0.1760 & -0.1569
\end{array}\right) \text {, } \\
& T_{16}=T_{17} \\
& =\left(\begin{array}{cccc}
-167.8742 & 206.0977 & 254.4031 & -44.6630 \\
15.0769 & 147.1908 & 106.7331 & -62.5135 \\
462.5231 & 174.7488 & -97.4980 & -165.1735 \\
532.2860 & 236.1899 & -93.3484 & -210.2411
\end{array}\right) \text {, } \\
& T_{26}=T_{27}=\left(\begin{array}{ccc}
-1.0162 & 0.5879 & -2.5760 \\
1.5155 & -0.8767 & 3.8419 \\
8.8346 & -5.1108 & 22.3961 \\
10.5625 & -6.1104 & 26.7763
\end{array}\right) \\
& T_{36}=T_{37} \\
& =\left(\begin{array}{cccc}
123.6493 & -224.3619 & -253.0593 & 60.0567 \\
59.3071 & -107.6129 & -121.3773 & 28.8056 \\
-26.7899 & 48.6103 & 54.8279 & -13.0119 \\
-19.6265 & 35.6123 & 40.1673 & -9.5326
\end{array}\right) .
\end{aligned}
$$


The coefficients in case of the uncertainty $\Delta_{1}$ are as follows:

$$
\begin{array}{r}
\lambda_{11}=0.33, \quad \lambda_{21}=0.33, \quad \lambda_{31}=0.34, \\
\lambda_{12}=0.4, \quad \lambda_{22}=0.3, \quad \lambda_{32}=0.3, \\
\lambda_{13}=0.6, \quad \lambda_{23}=0.4, \\
\lambda_{14}=0.45, \quad \lambda_{24}=0.55, \\
\lambda_{15}=0.33, \quad \lambda_{25}=0.37, \quad \lambda_{35}=0.4, \\
\lambda_{16}=0.35, \quad \lambda_{26}=0.36, \quad \lambda_{36}=0.39, \\
\lambda_{17}=0.41, \quad \lambda_{27}=0.3, \quad \lambda_{37}=0.39 .
\end{array}
$$

The coefficients in case of the uncertainty $\Delta_{2}$ are as follows:

$$
\begin{aligned}
& \lambda_{11}=0.1, \quad \lambda_{21}=0.2, \quad \lambda_{31}=0.7, \\
& \lambda_{12}=0.5, \quad \lambda_{22}=0, \quad \lambda_{32}=0.5 \text {, } \\
& \lambda_{13}=0, \quad \lambda_{23}=1 \text {, } \\
& \lambda_{14}=0.81, \quad \lambda_{24}=0.19 \text {, } \\
& \lambda_{15}=0.3, \quad \lambda_{25}=0.5, \quad \lambda_{35}=0.2, \\
& \lambda_{16}=0.02, \quad \lambda_{26}=0.13, \quad \lambda_{36}=0.85 \text {, } \\
& \lambda_{17}=0.3, \quad \lambda_{27}=0.1, \quad \lambda_{37}=0.6 .
\end{aligned}
$$

\section{Acknowledgment}

This work is supported by the National Natural Science Foundation of China under Grants 61074026 and 90916003.

\section{References}

[1] A. Jadbabaie, J. Lin, and A. S. Morse, "Coordination of groups of mobile autonomous agents using nearest neighbor rules," IEEE Transactions on Automatic Control, vol. 48, no. 6, pp. 988-1001, 2003.

[2] R. Olfati-Saber and R. M. Murray, "Consensus problems in networks of agents with switching topology and time-delays," IEEE Transactions on Automatic Control, vol. 49, no. 9, pp. 15201533, 2004.

[3] W. Ren and R. W. Beard, "Consensus seeking in multiagent systems under dynamically changing interaction topologies," IEEE Transactions on Automatic Control, vol. 50, no. 5, pp. 655661, 2005.

[4] P. Lin and Y. Jia, "Further results on decentralised coordination in networks of agents with second-order dynamics," IET Control Theory \& Applications, vol. 3, no. 7, pp. 957-970, 2009.

[5] G. Wen, Z. Duan, W. Yu, and G. Chen, "Consensus in multiagent systems with communication constraints," International Journal of Robust and Nonlinear Control, vol. 22, no. 2, pp. 170$182,2012$.

[6] Z. Li, Z. Duan, G. Chen, and L. Huang, "Consensus of multiagent systems and synchronization of complex networks: a unified viewpoint," IEEE Transactions on Circuits and Systems. I. Regular Papers, vol. 57, no. 1, pp. 213-224, 2010.

[7] P. Wieland, R. Sepulchre, and F. Allgöwer, "An internal model principle is necessary and sufficient for linear output synchronization," Automatica, vol. 47, no. 5, pp. 1068-1074, 2011.
[8] G. Wen, Z. Li, Z. Duan, and G. Chen, "Distributed consensus control for linear multi-agent systems with discontinuous observations," International Journal of Control, vol. 86, no. 1, pp. 95-106, 2013.

[9] W. Yu, G. Chen, and M. Cao, "Consensus in directed networks of agents with nonlinear dynamics," IEEE Transactions on Automatic Control, vol. 56, no. 6, pp. 1436-1441, 2011.

[10] W. Ren and N. Sorensen, "Distributed coordination architecture for multi-robot formation control," Robotics and Autonomous Systems, vol. 56, no. 4, pp. 324-333, 2008.

[11] Z. Li and Z. Duan, "Distributed adaptive attitude synchronization of multiple spacecraft," Science China Technological Sciences, vol. 54, pp. 1992-11998, 2011.

[12] G. Wen, Z. Duan, Z. Li, and G. Chen, "Consensus and its $\mathscr{L}_{2}$-gain performance of multi-agent systems with intermittent information transmissions," International Journal of Control, vol. 85, no. 4, pp. 384-396, 2012.

[13] G. Hu, "Robust consensus tracking of a class of second-order multi-agent dynamic systems," Systems \& Control Letters, vol. 61, no. 1, pp. 134-142, 2012.

[14] U. Münz, A. Papachristodoulou, and F. Allgöwer, "Delay robustness in non-identical multi-agent systems," IEEE Transactions on Automatic Control, vol. 57, no. 6, pp. 1597-1603, 2012.

[15] U. T. Jönsson and C.-Y. Kao, "Consensus of heterogeneous LTI agents," IEEE Transactions on Automatic Control, vol. 57, no. 8, pp. 2133-2138, 2012.

[16] H. Kim, H. Shim, and J. H. Seo, "Output consensus of heterogeneous uncertain linear multi-agent systems," IEEE Transactions on Automatic Control, vol. 56, no. 1, pp. 200-206, 2011.

[17] M. Ji, G. Ferrari-Trecate, M. Egerstedt, and A. Buffa, "Containment control in mobile networks," IEEE Transactions on Automatic Control, vol. 53, no. 8, pp. 1972-1975, 2008.

[18] Z. Li, W. Ren, X. Liu, and M. Fu, "Distributed containment control of multiagent systems with general linear dynamics in the presence of multiple leaders," International Journal of Robust and Nonlinear Control, vol. 23, no. 5, pp. 534-547, 2013.

[19] M. Porfiri, D. G. Roberson, and D. J. Stilwell, “Tracking and formation control of multiple autonomous agents: a two-level consensus approach," Automatica, vol. 43, no. 8, pp. 1318-1328, 2007.

[20] Q. Song, J. Cao, and W. Yu, "Second-order leader-following consensus of nonlinear multi-agent systems via pinning control," Systems \& Control Letters, vol. 59, no. 9, pp. 553-562, 2010.

[21] G. Chen and F. L. Lewis, "Coordination of networked systems on digraphs with multiple leaders via pinning control," International Journal of Systems Science, vol. 43, no. 2, pp. 368-384, 2012.

[22] G. Chen and F. L. Lewis, "Leader-following control for multiple inertial agents," International Journal of Robust and Nonlinear Control, vol. 21, no. 8, pp. 925-942, 2011.

[23] J. Huang, Nonlinear Output Reguation: Theory and Applications, vol. 8, SIAM, Philadelphia, Pa, USA, 2004.

[24] Y. Hong, X. Wang, and Z. P. Jiang, "Distributed output regulation of leader-follower multi-agent systems," International Journal of Robust and Nonlinear Control, vol. 23, no. 1, pp. 4866, 2013.

[25] X. Wang, Y. Hong, J. Huang, and Z.-P. Jiang, "A distributed control approach to a robust output regulation problem for multiagent linear systems," IEEE Transactions on Automatic Control, vol. 55, no. 12, pp. 2891-2895, 2010. 
[26] Y. Su and J. Huang, "Cooperative output regulation of linear multi-agent systems," IEEE Transactions on Automatic Control, vol. 57, no. 4, pp. 1062-1066, 2012.

[27] C. Godsil and G. F. Royle, Algebraic Graph Theory, SpringerVerlag, New York, NY, USA, 2001.

[28] F. Amato, Robust Control of Linear Systems Subject to Uncertain Time-Varying Parameters, Springer, Berlin, Germany, 2006.

[29] S. Dasgupta, C. H. Papadimitriou, and U. V. Vazirani, Algorithms, McGraw-Hill Science/Engineering/Math, New York, NY, USA, 2006. 


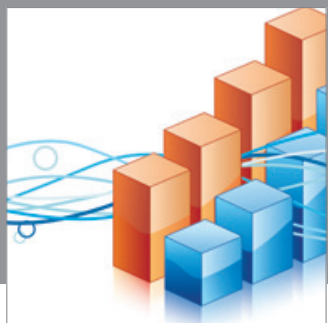

Advances in

Operations Research

mansans

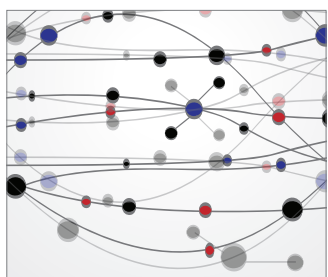

The Scientific World Journal
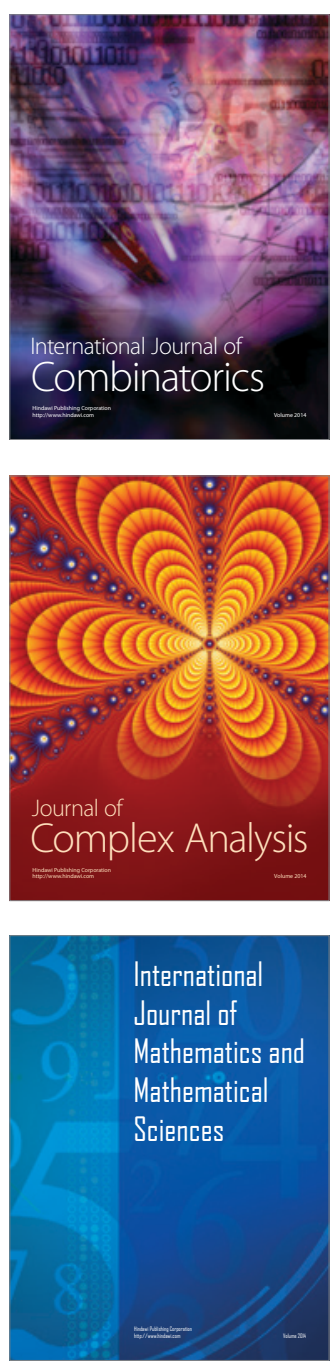
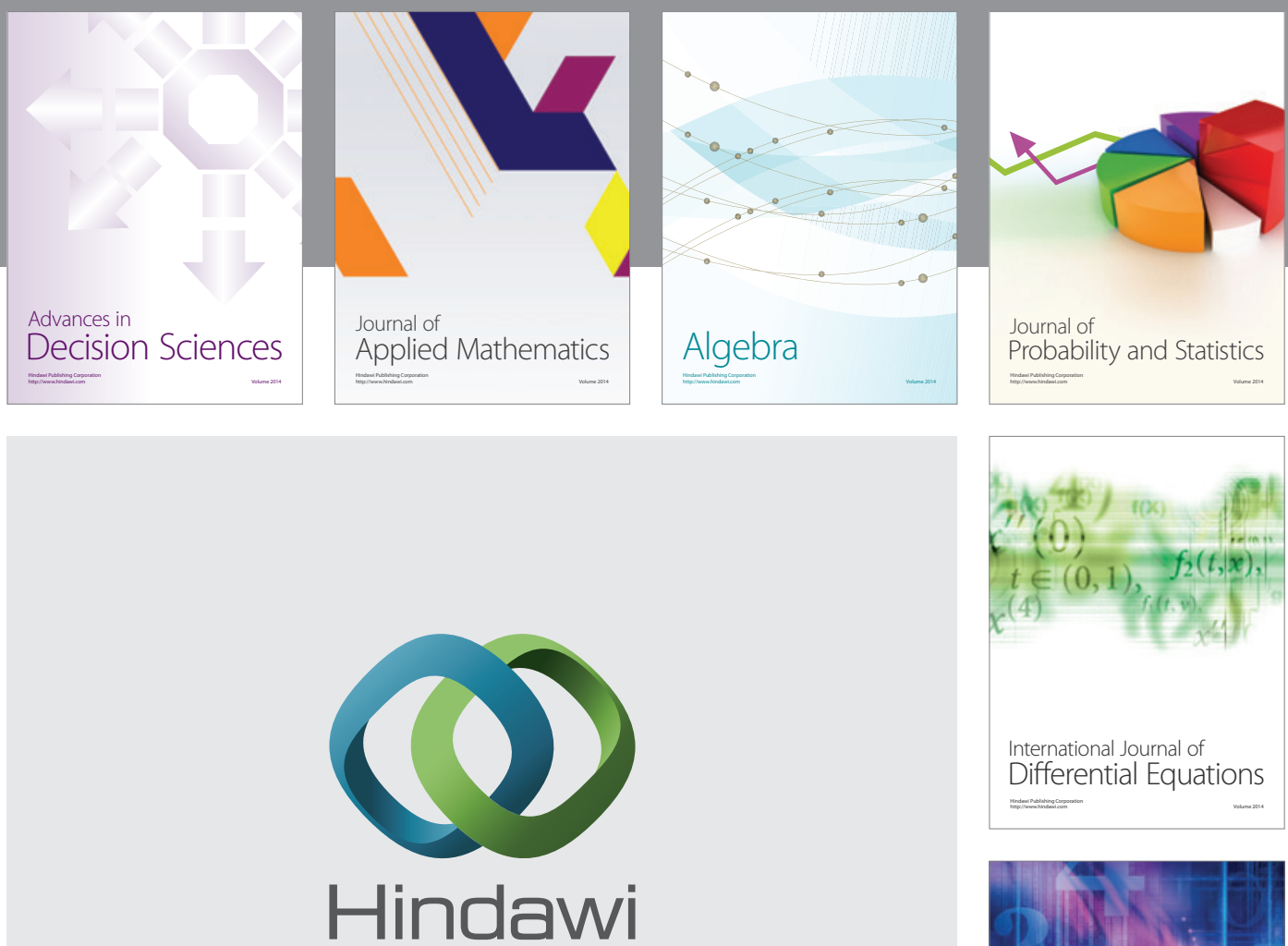

Submit your manuscripts at http://www.hindawi.com
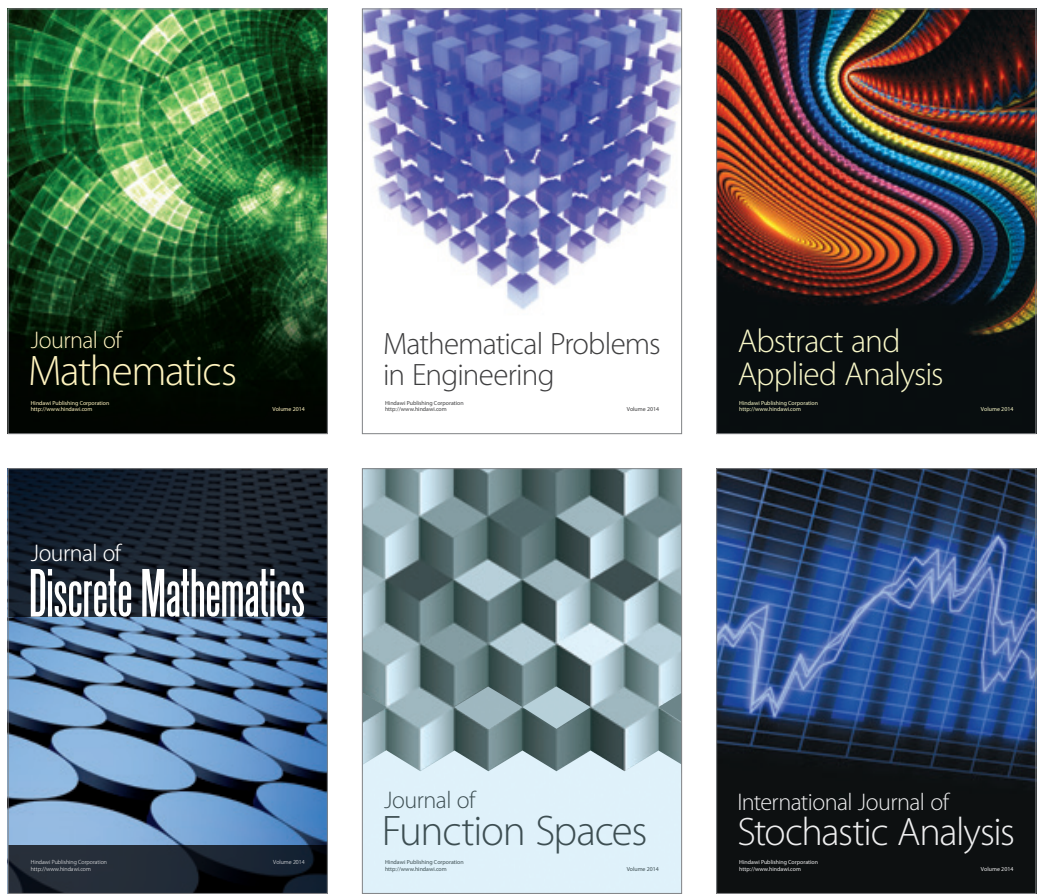

Journal of

Function Spaces

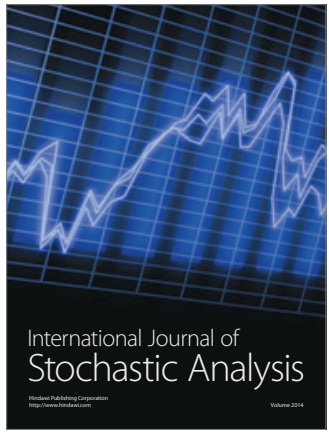

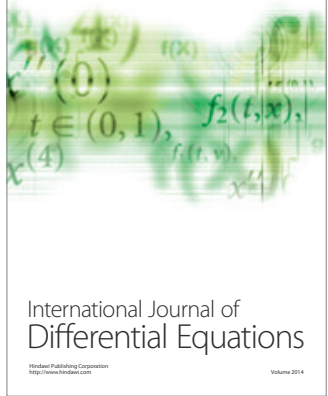
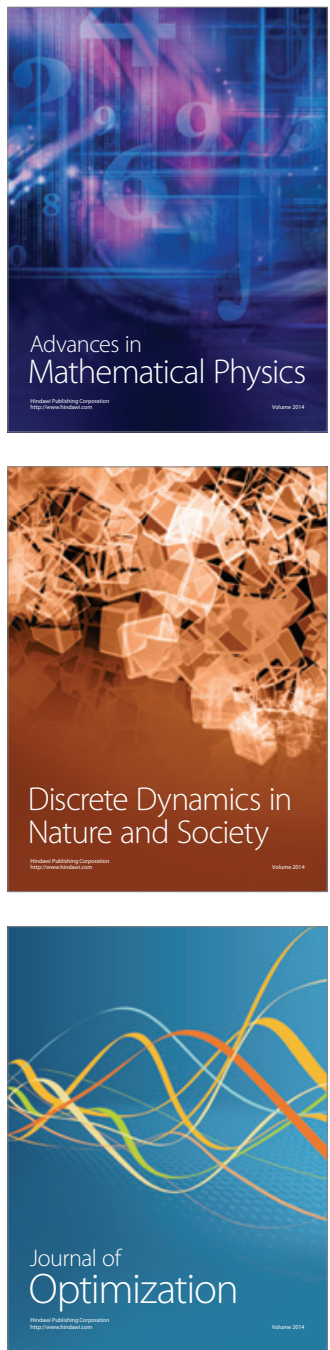